\title{
Effectiveness using higher inhaled corticosteroid dosage in patients with COPD by different blood eosinophilic counts
}

This article was published in the following Dove Press journal:

International Journal of COPD

21 September 2016

Number of times this article has been viewed

\author{
Shih-Lung Cheng ${ }^{1,2}$ \\ Ching-Hsiung Lin ${ }^{3-5}$ \\ 'Department of Internal Medicine, \\ Far Eastern Memorial Hospital, \\ Taipei, ${ }^{2}$ Department of Chemical \\ Engineering and Materials Science, \\ Yuan Ze University, Zhongli, \\ Taoyuan City, ${ }^{3}$ Department of \\ Internal Medicine, Division of Chest \\ Medicine, Changhua Christian \\ Hospital, Changhua, ${ }^{4}$ Department of \\ Respiratory Care, College of Health \\ Sciences, Chang Jung Christian \\ University, Tainan, ${ }^{5}$ School of Medicine, \\ Chung Shan Medical University, \\ Taichung City, Taiwan
}

Background: Blood eosinophil counts have been documented as a good biomarker for patients with chronic obstructive pulmonary disease (COPD) using inhaled corticosteroid (ICS) therapy. However, the effectiveness and safety of prescribing high or medium dose of ICS for patients with different eosinophil counts are unknown.

Methods: A post hoc analysis of a previous prospective randomized study was performed for COPD patients using higher dose (HD: Fluticasone 1,000 $\mu \mathrm{g} /$ day) or medium dose (MD: Fluticasone $500 \mu \mathrm{g}$ /day) of ICS combined with Salmeterol ( $100 \mu \mathrm{g}$ /day). Patients were classified into two groups: those with high eosinophil counts ( $\mathrm{HE} \geq 3 \%$ ) and those with low eosinophil counts (LE $<3 \%$ ). Lung function was evaluated with forced expiratory volume in 1 second, forced vital capacity, and COPD assessment test. Frequencies of acute exacerbation and pneumonia were also measured.

Results: Two hundred and forty-eight patients were studied and classified into higher eosinophil (HE) (n=85, 34.3\%) and lower eosinophil (LE) groups ( $\mathrm{n}=163,65.7 \%)$. The levels of forced expiratory volume in 1 second were significantly increased in patients of HE group treated with HD therapy, compared with the other groups (HE/HD: 125.9 $\pm 27.2 \mathrm{~mL}$ vs HE/MD: 94.3 $\pm 23.7 \mathrm{~mL}$, vs LE/HD: $70.4 \pm 20.5 \mathrm{~mL}$, vs LE/MD: $49.8 \pm 16.7 \mathrm{~mL} ; P<0.05$ ) at the end of the study. Quality of life (COPD assessment test) markedly improved in HE/HD group than in MD/LE group (HE/HD: $9 \pm 5$ vs LE/MD: $16 \pm 7, P=0.02$ ). The frequency of acute exacerbation was more decreased in HE/HD group patients, compared with that in LE/MD group (HE/HD: $13.5 \%$ vs LE/MD: $28.7 \%, P<0.01)$. Pneumonia incidence was similar in the treatment groups (HE/HD: 3.2\%, HE/MD: 2.6\%, LE/HD: 3.5\%, LE/MD 2.8\%; $P=0.38$ ).

Conclusion: The study results support using blood eosinophil counts as a biomarker of ICS response and show the benefits of greater improvement of lung function, quality of life, and decreased exacerbation frequency in COPD patients with blood eosinophil counts higher than $3 \%$, especially treated with higher dose of ICS.

Keywords: chronic obstructive pulmonary disease, inhaled corticosteroid, blood eosinophil

\section{Introduction}

Inhaled corticosteroids (ICSs) reduce the risk of moderate and severe chronic obstructive pulmonary disease (COPD) acute exacerbation (AE) in patients with moderate to very severe lung function defect and a history of frequent exacerbation. ${ }^{1-5}$ However, treatment benefits are slight and are offset by an increased risk of adverse effects, especially pneumonia. ${ }^{6}$ Therefore, identification of a simple biomarker associated with treatment response would be necessary. Airway eosinophil inflammation, a significant feature of asthma, is recognized as an inflammatory endotype in COPD. ${ }^{7-9}$ COPD with eosinophilic
Correspondence: Ching-Hsiung Lin Department of Internal Medicine, Division of Chest Medicine, Changhua Christian Hospital, I 35 South School

Street, Changhua 500, Taiwan

Tel +88647238595

Fax +886 47228289

Email teddy@cch.org.tw (c) (1) (3) 2016 Cheng and Lin. This work is published and licensed by Dove Medical Press Limited. The full terms of this license are available at https://www.dovepress.com/terms.php
and incorporate the Creative Commons Attribution - Non Commercial (unported, 33.0$)$ License (http://creativecommons.org/licenses/by-nd/3.0/). By accessing the work you

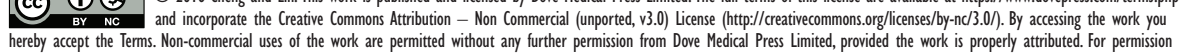
hereby accept the Terms. Non-commercial uses of the work are permitted without any further permission from Dove Medis
for commercial use of this work, please see paragraphs 4.2 and 5 of our Terms (https://www.dovepress.com/terms.php). 
inflammation, defined as sputum eosinophils $\geq 3 \%$, is reported to be found in up to $28 \%$ of cases during AEs, ${ }^{10}$ and interestingly, it is seen in approximately $34 \%{ }^{11}$ (or $38 \%{ }^{12}$ ) of COPD patients in periods of stable disease. Airway eosinophilic inflammation is a reliable predictor of benefit from inhaled and oral corticosteroid treatment in patients with COPD. ${ }^{12-15}$

The measurement and evaluation of airway eosinophilic inflammation usually require the assessment of induced sputum examination. ${ }^{8}$ Sputum induction is a direct and reliable method of evaluating airway inflammation characteristics. However, there exist a number of limitations. ${ }^{16,17}$ In addition to being unsuitable for point-of-care testing, it requires more experience to differentiate inflammatory counts and may not be always precise and successful (failure rate near $30 \%) .{ }^{16,17}$ Due to these reasons, alternative tools for minimally invasive and easily applicable diagnostic methods that can detect sputum eosinophilia in asthma and COPD have been emphasized. ${ }^{10,11,18-21}$ The use of peripheral serum cell counts may be a potential alternative and attracting tool due to its ease of application in our daily practice. The association of sputum eosinophilia and blood eosinophils in asthma patients has been demonstrated, with promising results. ${ }^{21-24}$ In COPD patients, however, very few studies have mentioned this blood eosinophils application tool, particularly in clinical stability for these patients. A recent study in 20 COPD patients and 21 healthy controls has reported the relationship between bronchial and blood eosinophil counts. ${ }^{25}$ Studies have also demonstrated that the blood eosinophil counts may serve as a biomarker of corticosteroid treatment effectiveness in exacerbating ${ }^{26}$ and stable ${ }^{27,28}$ COPD patients. The clinical characteristics of stable COPD patients with higher levels of blood eosinophils ( $\geq 2 \%$ ) and their series changes occurring during a 3-year follow-up have also been elucidated. ${ }^{9}$ Therefore, blood eosinophil counts seem to be a reliable biomarker to predict exacerbation status and ICS-treated responsiveness in COPD patients.

Our previous study had documented that COPD patients treated with higher ICS dose have more clinical effectiveness compared to those treated with medium ICS dose. ${ }^{29}$ However, the benefits and safety of high or medium dose for patients with different blood eosinophil counts are unknown. The aim of the study was to compare the treatment effectiveness of different ICS doses in COPD patients with different blood eosinophilic counts.

\section{Methods}

\section{Study design}

Ethical approval was received from the institutional review board/ethics committee of Far Eastern Memorial hospital.
Written informed consent was obtained from all study subjects. This study was a post hoc analysis of our previous study ${ }^{29}$ in which the patients were nonblinded and randomized 1:1 to one of two treatment groups: high-dose (HD) and medium-dose (MD) ICS groups. From the previous drug therapy, these COPD patients were randomized to and prescribed one of the two treatment doses, which were higher dosage (HD) and medium dosage (MD) of ICS. The definition of higher dose of ICS was Fluticasone $1,000 \mu \mathrm{g} /$ day and medium dose of ICS was Fluticasone $500 \mu \mathrm{g} /$ day. Both treatment regimens were combined with Salmeterol $100 \mu \mathrm{g} /$ day and were administered by a hydrofluoroalkane pressurized metered-dose inhaler with a space device (GlaxoSmithKline plc, London, UK).

\section{Characteristics of the participants}

The diagnoses of these participants were according to the following criteria: history, physical examination, chest radiograph findings, and spirometric data. Inclusion criteria for COPD were as follows: chronic airway signs and symptoms such as cough with sputum, breathlessness, wheezing, and confirmed chronic airway obstruction, which was defined as 1) forced expiratory volume in 1 second $\left(\mathrm{FEV}_{1}\right) /$ forced vital capacity (FVC) $<70 \%$ and 2 ) $\mathrm{FEV}_{1}<80 \%$ of the predicted value by spirometric data, and $\mathrm{FEV}_{1}$ reversibility after inhalation of $200 \mu \mathrm{g}$ salbutamol of $<12 \%$ of prebronchodilator $\mathrm{FEV}_{1}$. Men and women (aged over 40 years) with COPD diagnosed for over 6 months prior to screening were enrolled in the study. Smoking history with at least 10 pack-years should be noted.

Patients with the following conditions were excluded: AE of life-threatening COPD within the past year, hospitalization or emergency department visit for COPD in the 4 weeks prior to screening period, systemic corticosteroid used in the month before screening, a history of nonsmoking or smoking an equivalent to $<10$ pack-years, significant asthma diagnosis with airway reversible responsibility, active pulmonary tuberculosis disease, and clinically significant respiratory tract infection in the 4 weeks before screening. Current use of medications that would have an effect on bronchospasm and/or lung function was also a criterion for exclusion.

\section{Study protocol}

For this post hoc analysis of our randomized study, patients were classified into two groups according to their blood eosinophil counts: high eosinophil group with $\geq 3 \%$ eosinophil count (HE) and low eosinophil group with $<3 \%$ eosinophil count (LE). The cutoff point of 3\% eosinophil count was the reference, and therefore, sputum eosinophil 
counts over $3 \%$ will be of greater benefit with steroid therapy. ${ }^{13}$ Therefore, patients were divided into having one of the four conditions, HE/HD, HE/MD, LE/HD, and LE/MD, respectively, according to their blood eosinophil counts (HE, LE) and ICS doses (HD, MD). The assessment parameters included lung function tests by spirometry $\left(\mathrm{FEV}_{1}\right.$ and $\left.\mathrm{FVC}\right)$ and symptom scores using COPD assessment test (CAT) tool. ${ }^{30}$ Besides, the frequency of AE (COPD deterioration resulting in emergency treatment, hospitalization, or treatment with additional systemic steroid or antibiotic agents) was also measured. Safety issues were also surveyed, such as pneumonia incidence.

\section{Data analysis}

Categorical variables were compared using chi-square test, and continuous variables were compared by Student's $t$-test. A $P$-value of $<0.05$ was considered significant. Fisher's exact test (qualitative data) or analysis of variance (quantitative data) was performed to test the homogeneity of the treatment groups. A Hosmer-Lemeshow goodness-of-fit test was used to help determine if the model fit to the data well. All statistical analyses were performed using Statistical Package for the Social Sciences for Windows (version 9.0; SPSS Inc., Chicago, IL, USA).

\section{Results}

In the post hoc analysis, 248 patients were studied and classified as follows: 85 patients $(34.3 \%)$ with high eosinophil counts (HE group, $\geq 3 \%$ ) and 163 patients (65.7\%) with low eosinophil counts (LE group, <3\%) (Figure 1). The demographics and COPD characteristics of patients in the HE and LE groups are summarized in Table 1. Most patients $(84.9 \%)$ were male, and the average lung function was classified based on the Global initiative for chronic Obstructive Lung Disease III status (50.3\% of predicted in the HE group and $52.5 \%$ of predicted in the LE group). There was no significant difference between the two groups with respect to treatment with higher ICS or medium ICS doses $(P=0.39)$. Besides, regarding the lung function parameter with positive bronchodilator test responsiveness, there was no statistically significant difference between patients with high eosinophil counts and low counts (HE: $22.2 \%$ vs LE: $14.5 \%, P=0.08$ ). However, there was higher AE frequency in the previous 1 year in HE patients compared with LE patients (HE: 27.1\% vs LE: $7.4 \%, P<0.01)$.

Analysis of the pulmonary function status with $\mathrm{FEV}_{1}$ and FVC revealed that patients showed improvements in lung function across both treatment groups during the study. The improved $\mathrm{FEV}_{1}$ levels among these patients were as follows: HE/HD: $125.9 \pm 27.2 \mathrm{~mL}$ versus HE/MD: 94.2 $\pm 23.7(P<0.05)$, versus LE/HD: $70.4 \pm 20.5(P=0.03)$, and versus LE/MD: $49.8 \pm 16.7(P<0.01)$. The levels of FVC were as follows: HE/HD: $248.4 \pm 51.3 \mathrm{~mL}$ versus HE/MD: 198.6 $\pm 48.1(P<0.05)$, versus LE/HD: $145.2 \pm 47.5(P=0.02)$, and versus LE/MD: $82.6 \pm 44.3(P<0.01)$. There was more significant lung function improvement in $\mathrm{HE} / \mathrm{HD}$ patients when compared to that in patients of other groups (Figures 2 and 3). Patients receiving higher doses of Fluticasone showed greater efficacy in improvement of lung function, especially the patients also having high eosinophil counts.

Patient-assessed COPD symptoms scores with CAT questionnaire decreased in both HE and LE groups, with a statistically significant difference found in favor of the patients treated in the HD group. The CAT scores were as follows: HE/HD: 9 \pm 5 , HE/MD: $12 \pm 6$, LE/HD: $13 \pm 5$, and LE/MD: $16 \pm 7$ throughout the study (Figure 4). There was a trend of a higher quality of life among the patients who received continuous treatment with higher dose of ICS, especially in patients with accompanying high eosinophil counts, and statistically more significant difference in CAT scores among patients treated with medium ICS dose, even

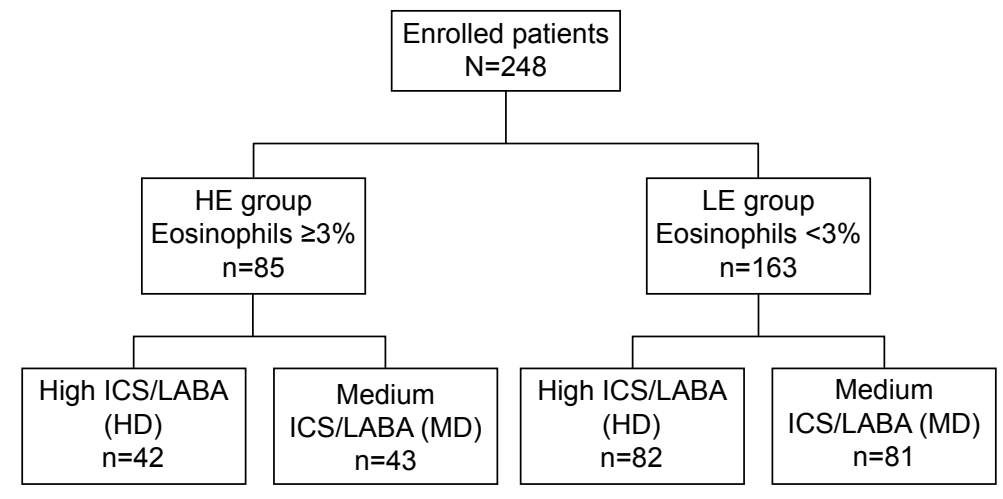

Figure I A flow chart for the number of subjects.

Abbreviations: HD, high dose; HE, higher eosinophil count; ICS, inhaled corticosteroid; LE, lower eosinophil count; MD, medium dose. 
Table I Baseline demographic characteristics of the study subjects with COPD

\begin{tabular}{|c|c|c|c|}
\hline Parameters & Eosinophil $\geq 3 \%$ (HE, $n=85)$ & Eosinophil $<3 \%$ (LE, $n=163)$ & $P$-value \\
\hline Age, mean $\pm S D$ & $68.7 \pm 19.2$ & $70.5 \pm 21.4$ & 0.46 \\
\hline $\operatorname{Sex}(M / F)$ & $69 / 16$ & $143 / 20$ & 0.75 \\
\hline Smoking (pack-years), mean \pm SD & $27.4 \pm 16.5$ & $29.1 \pm 19.3$ & 0.52 \\
\hline $\mathrm{AE}\left(>\mathrm{I}\right.$ admission), mean $\pm S D^{\mathrm{a}}$ & $23(27.1 \%)$ & $12(7.4 \%)$ & $0.01 *$ \\
\hline \multicolumn{4}{|l|}{ Lung function test } \\
\hline Bronchodilator test response $(+), \mathrm{n}(\%)$ & $19(22.2 \%)$ & $24(14.5 \%)$ & 0.08 \\
\hline Mild obstructive, $\mathrm{n}(\%)$ & 0 & $12(7.4 \%)$ & 0.31 \\
\hline Moderate obstructive, n (\%) & 45 (52.9\%) & $92(56.4 \%)$ & \\
\hline Severe obstructive, $\mathrm{n}(\%)$ & $29(34.1 \%)$ & $4 \mathrm{I}(25.2 \%)$ & \\
\hline Very severe obstructive, $\mathrm{n}(\%)$ & $11(12.9 \%)$ & $18(11.0 \%)$ & \\
\hline $\mathrm{FEV}_{1}(\mathrm{~L}), \mathrm{n}$ (\% of predicted value) & $1.29(50.3 \%)$ & $1.32(52.5 \%)$ & 0.32 \\
\hline FVC (L), n (\% of predicted value) & $2.36(60.4 \%)$ & $2.45(61.7 \%)$ & 0.41 \\
\hline $\mathrm{FEV}_{\mathrm{I}} / \mathrm{FVC}$ (\% of predicted value), mean $\pm \mathrm{SD}$ & $48.1 \pm 20.3$ & $50.6 \pm 22.9$ & 0.14 \\
\hline FEV , reversibility (\%), mean \pm SD & $8.3 \pm 5.1$ & $9.7 \pm 7.4$ & 0.44 \\
\hline CAT baseline, mean \pm SD & $18 \pm 6$ & $19 \pm 7$ & 0.37 \\
\hline \multicolumn{4}{|l|}{ Medications } \\
\hline Higher ICS/LABA (HD), n (\%) & $42(49.4 \%)$ & $82(50.3 \%)$ & 0.39 \\
\hline Medium ICS/LABA (MD), n (\%) & $43(50.6 \%)$ & $81(49.7 \%)$ & \\
\hline \multicolumn{4}{|l|}{ Comorbidity } \\
\hline HTN, n (\%) & $19(22.4 \%)$ & 31 (19.0\%) & 0.26 \\
\hline DM, n (\%) & $14(16.7 \%)$ & $26(15.9 \%)$ & \\
\hline CAD, n (\%) & 7 (8.2\%) & $16(9.8 \%)$ & \\
\hline $\mathrm{CHF}, \mathrm{n}(\%)$ & $9(10.6 \%)$ & $28(17.2 \%)$ & \\
\hline
\end{tabular}

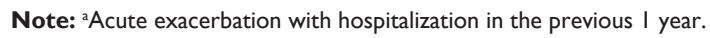

Abbreviations: AE, acute exacerbation; COPD, chronic obstructive pulmonary disease; CAT, COPD assessment test; $F$, female; FEV , forced expiratory volume in I second; FVC, forced vital capacity; HD, high dose; HE, higher eosinophil count; ICS, inhaled corticosteroid; M, male; MD, medium dose; LE, low eosinophil counts; HTN, hypertension; DM, Diabetes Mellitus; CAD, coronary artery disease; CHF, congestive heart failure; SD, standard deviation.

in those with high eosinophil counts (LE/HD). Average changes from baseline in the mean scores of CAT decrease also showed significant differences between $\mathrm{HE}$ patients and LE patients ( $8 \pm 3$ vs $4 \pm 2, P=0.04$ ) at the end of study, irrespective of whether the patients were treated with HD or MD therapy. There was greater benefit and more treatment response, especially in HE patients using ICS therapy.

Also, the frequency of AE detection showed a significant difference in the patients of all four groups. The percentage

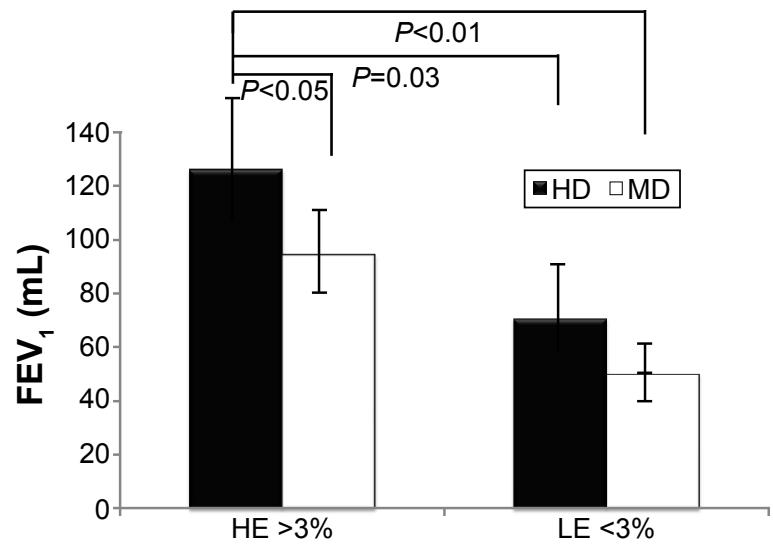

Figure 2 Lung function improvement with $\mathrm{FEV}_{\text {, }}$ in $\mathrm{HE}$ and LE patients using $\mathrm{HD}$ and MD therapy.

Abbreviations: $\mathrm{FEV}_{1}$, forced expiratory volume in I second; HD, high dose; $\mathrm{HE}$, higher eosinophil count; LE, lower eosinophil count; MD, medium dose. of $\mathrm{AE}$ after ICS/long-acting beta-agonist treatment was $\mathrm{HE} /$ HD: 0.135, HE/MD: 0.147, LE/MD: 0.226, and LE/MD: 0.287 per person-year, respectively (Figure 5). There was a significant difference and reduction in the percentage of $\mathrm{AE}$ in HE patients treated with HD, compared with other patients. None of the patients suffered from life-threatening AE. Meanwhile, there was no obvious difference in the incidence of pneumonia in these groups (HE/HD: 3.2\%, HE/MD: 2.6\%, LE/HD: $3.5 \%$, LE/MD 2.8\%; $P=0.38$ ) (Figure 6).

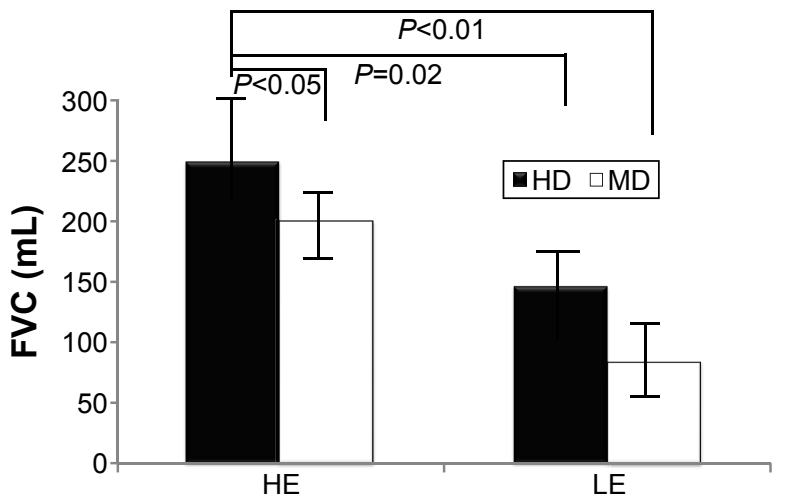

Figure 3 Lung function improvement with FVC in HE and LE patients using HD and MD therapy.

Abbreviations: FVC, forced vital capacity; HD, high dose; HE, higher eosinophil count; LE, lower eosinophil count; MD, medium dose. 


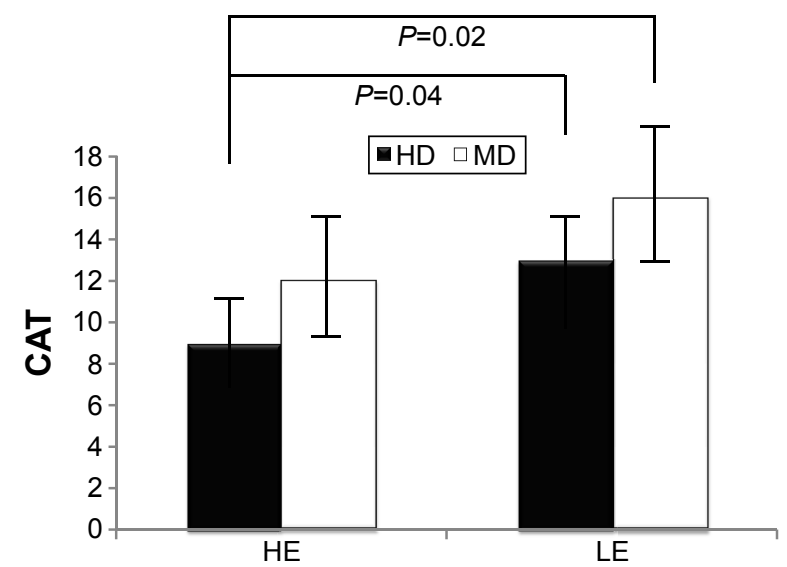

Figure 4 Quality of life improvement with CAT scores in HE and LE patients using $\mathrm{HD}$ and MD therapy.

Abbreviations: CAT, COPD assessment test; HD, high dose; HE, higher eosinophil count; LE, lower eosinophil; MD, medium dose.

Other side effects such as hoarseness or oral candidiasis were similar among the patients in the four groups (HE/HD: 6.3\%, HE/MD: 8.8\%, LE/HD: 4.9\%, LE/MD 9.2\%; $P=0.38)$.

\section{Discussion}

Our post hoc analysis demonstrated that the responsiveness was greater in lung function, quality of life, and decreased frequency of $\mathrm{AE}$ among COPD patients with high blood eosinophil count ( $\geq 3 \%$ ) undergoing ICS treatment, especially in those on high-dose ICS therapy. The results were similar to other studies ${ }^{26-28}$ in which greater effectiveness was noticed in treating high eosinophil counts with ICS. However, this is the first study to compare the benefits shown by high and medium ICS dose in COPD patients with high eosinophil count. Results of this study showed greater improvement from treatment with high ICS dose (Fluticasone $1,000 \mu \mathrm{g} /$ day) than with medium ICS dose (Fluticasone $500 \mu \mathrm{g} /$ day). Therefore, it is helpful to select the most

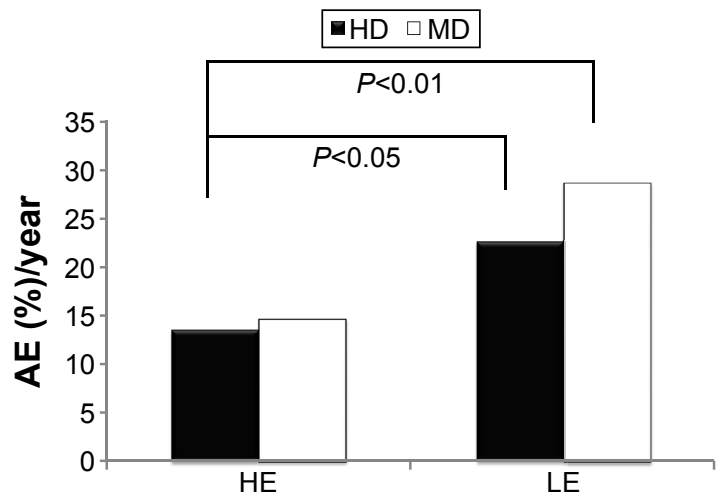

Figure 5 Frequency of annual acute exacerbation given as percentage in $\mathrm{HE}$ and LE patients using HD and MD therapy.

Abbreviations: $A E$, acute exacerbation; $H D$, high dose; $H E$, higher eosinophil count; LE, lower eosinophil count; MD, medium dose.

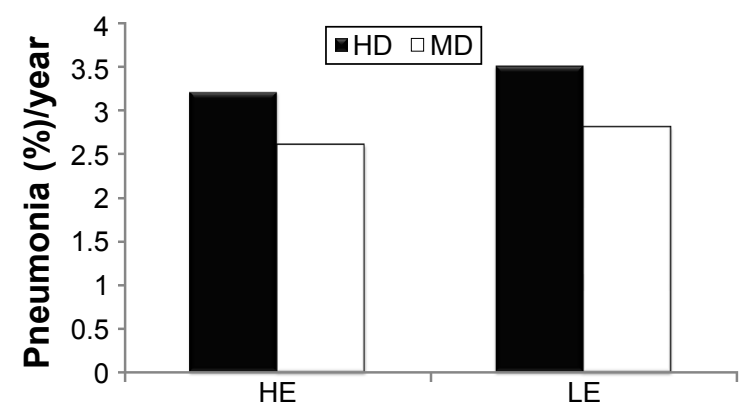

Figure 6 Incidence of annual pneumonia given as percentage in HE and LE patients using HD and MD therapy.

Abbreviations: HD, high dose; HE, higher eosinophil count; LE, lower eosinophil count; MD, medium dose.

appropriate treatments for individual patients and the blood eosinophil count has greater potential as a biomarker to improve decision making in COPD treatment.

Blood eosinophil count, a promptly available and easy-tointerpret measure, is probably a suitable tool as a biomarker in clinical practice to identify which COPD patients are most likely to benefit from the treatment efficacy of ICS. Our study revealed that there were higher exacerbation and hospitalization rates in patients with high eosinophil counts than in those with low eosinophil counts. Our result is similar to a recent report in which increased blood eosinophil levels above $0.34 \times 10^{9}$ cells $/ \mathrm{L}$ were associated with a 1.76 -fold increased risk of severe exacerbations. ${ }^{31}$ Therefore, it may have immediate clinical implications as there are ongoing more complex inflammation processes, such as asthma pathogenesis, in the airway in patients with high eosinophil counts and the benefits were greater with the use of ICS treatment in patients with extreme higher eosinophil counts ${ }^{27,28}$ or with the use of high ICS dose to reduce antiallergic inflammation in these patients. A recent study, ${ }^{32}$ however, reported that eosinopenia is a marker of poor outcome in COPD AE. It is important to clarify the true impact of the role of and the inflammation mechanism of eosinophils in patients with COPD.

The threshold for high eosinophil counts in COPD patients was not confirmed. Several studies ${ }^{26,27,33}$ had used $2 \%$ as the cutoff for the blood eosinophil level and documented that for patients with over $2 \%$ eosinophils, ICS combined with long-acting beta-agonist was associated with significant reduction in exacerbation rates versus Tiotropium (INSPIRE study ${ }^{34}$ ) or placebo (TRISTAN study). ${ }^{35}$ Stratification by blood eosinophil level $\geq 3 \%$ versus $<3 \%$ showed similar trends for TRISTAN. In the Foster 48-Week Trial to Reduce Exacerbation in COPD (FORWARD) study, ${ }^{28}$ the stratified method involved total eosinophil counts, not the percentage. In our study, the threshold of 3\% eosinophil level had shown significant difference in previous AE rate and a good response 
to high-dose therapy of ICS. The definition using 3\% level in blood was based on the sputum cutoff level ${ }^{10-12}$ in our study. It was found that the 3\% cutoff level was associated with increased exacerbation risk than the $2 \%$ threshold level. Also, the clinical effectiveness with ICS was greater in patients with $\geq 3 \%$ than in those with $<3 \%$, but there was no significant difference if the threshold level was $2 \%$. It should be further elucidated and studied as to which classifications of eosinophil levels are beneficial to the treatment guide.

A recent study had mentioned that patients with higher sputum eosinophils can predict more bronchial hyperresponsiveness (BHR) than the patients without $\mathrm{BHR}$, and those with sputum eosinophils $\geq 3 \%$ had more frequent exacerbations in the previous year and higher symptom scores than the patients with sputum eosinophils $<3 \%$. ${ }^{36}$ Sputum eosinophil count is known to be the most reliable predictor of responsiveness to ICS in COPD. However, there was no association between BHR and blood eosinophil percentage in our study, which is similar to another report. ${ }^{37}$ The different sites of eosinophil counts may represent variable clinical characteristics. This phenomenon is like that reported in a recent study on uncontrolled asthma patients, of whom one third exhibited inconsistency between sputum and blood eosinophils. ${ }^{38}$ There are some studies that confirm the concordance or discordance between sputum and blood eosinophils in patients with COPD. It should be further elucidated whether sputum eosinophil levels are in concordance with blood eosinophil counts or not, and airway eosinophil inflammation is compatible or not with systemic eosinophil inflammation in COPD.

Possible mechanisms for the inconsistency in blood and sputum eosinophils may consider the imbalance between the production by allergy and the subsequent clearance of eosinophils by airway macrophage ${ }^{39}$ and various stimulants in the inflammatory process of eosinophil recruitment into the airways. ${ }^{38}$ The cause for only a small group of the COPD patients exhibiting higher blood eosinophils may be that it may be a response to the association of several kinds of biological mechanisms underlying eosinophilic inflammation in the pathogenesis (endotypes) and clinical characteristics (phenotype) of COPD. ${ }^{40}$ Similar to the pathogenesis of asthma, stimulation and subsequent production of $\mathrm{T}$ helper (Th)2 cytokines may be responsible for eosinophilic inflammation in COPD subgroup. ${ }^{40}$ However, it has also been reported that the coexisting eosinophilic inflammation in the airway of COPD patients is not due to elevated levels of Th2 cytokines responsiveness. ${ }^{41}$ This may result from the non-Th2 eosinophilic inflammation in COPD, which is induced by the epithelial innate lymphoid cell type 2 pathways, and innate lymphoid cell type 2 has been documented the relationship of pathogenesis in severe nonallergic eosinophilic asthma. ${ }^{42}$ Therefore, further studies should be performed in order to elucidate the pathogenesis involved in the association between different endotypes of eosinophil inflammation and COPD phenotypes.

Based on these inflammatory mechanisms, the role of ICS in suppressing eosinophilic inflammation is rational and essential. More importantly, there was more clinical effectiveness when HE patients were treated with ICS therapy, especially with a high ICS dose. There is still a controversy regarding the benefits and risks of ICS therapy in COPD patients. Our current and previous findings show that high ICS doses have greater clinical benefits in lung function improvement, improvement of quality of life, and reduction of AE, especially in the HE subset of COPD patients. The side effects, especially the incidence of pneumonia, did not increase in patients on high ICS therapy. Therefore, our results provide a reliable and effective strategy of prescribing high ICS therapy in COPD patients with high eosinophil count.

\section{Limitations}

The major limitation and weakness of the study is the post hoc analysis nature of the analyses, although the rationale for the study was based on previous findings. This survey is from our randomized controlled trial and appropriate analyses were put in place to control for confounding factors. Indeed, the designed parameters and factors for this survey are not the primary endpoints originally. The second limitation is the difficultly to assess blood eosinophilia as the only marker for measurement of ICS efficacy; also, we chose the cutoff point $\geq 3 \%$ to divide the groups, based on a previous study performed on sputum eosinophilia. There is no clear data to support that blood eosinophilia reflects sputum eosinophilia in patients with COPD. The strong basis to test the value of blood eosinophils as a marker to ICS treatment and comparison between the treatment effectiveness of high and medium dose of ICS prompted us to do this elucidation. Further prospective studies should be carried out with large sample sizes to highlight the usefulness and reliability of eosinophil counts as a biomarker in COPD patients.

\section{Conclusion}

In conclusion, COPD patients with high eosinophils ( $\geq 3 \%$ ) treated with high doses of ICS had improved lung function status, decreased exacerbation frequency, and showed 
greater relief from symptoms in comparison to those using medium doses of ICS and to other low-eosinophil $(<3 \%)$ subgroups. Besides, no significant increase in the incidence of pneumonia development, especially on using high doses of ICS, was noticed. The study was the first study to compare the effectiveness of medium and high ICS doses in treatment of COPD with different eosinophil counts and provide an applicable dose for anti-inflammation response in COPD.

\section{Acknowledgment}

The abstract of this paper was presented at the American Thoracic Society International Conference, San Francisco, CA, USA, on May 13-18, 2016, as a poster presentation with interim findings. The poster's abstract was published in American Thoracic Society International Conference Abstracts, C103. Eosinophils in COPD and the asthma-COPD overlap syndrome: sorting through the chaos of ACOS, 2016, pp. A6243. This study was supported by a grant from the Far Eastern Memorial Hospital (FEMH 2016-D-011).

\section{Disclosure}

The authors report no conflicts of interest in this work.

\section{References}

1. Ferguson GT, Anzueto A, Fei R, Emmett A, Knobil K, Kalberg C. Effect of fluticasone propionate/salmeterol (250/25 microg) or salmeterol (50 microg) on COPD exacerbation. Respir Med. 2008; 102(8):1099-1108.

2. Dransfield MT, Bourbeau J, Jones PW, et al. Once-daily inhaled fluticasone furoate and vilanterol versus vilanterol only for prevention of exacerbation of COPD: two replicate double-blind, parallel-group, randomized controlled trial. Lancet Respir Med. 2013;1(3):210-223.

3. Szafranski WW, Cukier A, Ramirez A, et al. Efficacy and safety of budesonide/formoterol in the management of chronic obstructive pulmonary disease. Eur Respir J. 2003;21(1):74-81.

4. Sharafkhaneh A, Southard JG, Goldman M, Uryniak T, Martin UJ. Effect of budesonide/formoterol pMDI on COPD exacerbation: a double-blind, randomized study. Respir Med. 2012;106(2):257-268.

5. Agarwai R, Aggarwai AN, Gupta D, Jindal SK. Inhaled corticosteroids vs placebo for preventing COPD exacerbations: a systemic review and metaregression of randomized controlled trials. Chest. 2010;137(2): 318-325.

6. Suissa S. Number needed to treat in COPD: exacerbations versus pneumonias. Thorax. 2013;68(9):540-543.

7. Saha S, Brightling CE. Eosinophilic airway inflammation in COPD. Int J Chron Obstruct Pulmon Dis. 2006;1(1):39-47.

8. Brightling CE. Clinical applications of induced sputum. Chest. 2006;129(5):1344-1348.

9. Singh D, Kolsum U, Bringtling CE, Locantore N, Agusti A, Tsl-Singer R; for ECLIPSE invertigators. Eosinophilic inflammation in COPD: prevalence and clinical characteristics. Eur Respir J. 2014; 44(6):1697-1700.

10. Bafadhel M, Mckenna S, Terry S, et al. Acute exacerbations of chronic obstructive pulmonary disease: identification of biologic clusters and their biomarkers. Am J Respir Crit Care Med. 2011;184(6): $662-671$.
11. McDonald VM, Higgins I, Wood LG, Gibson PG. Multidimensional assessment and tailored interventions for COPD: respiratory utopia or common sense? Thorax. 2013;68(7):691-694.

12. Leigh R, Pizzichini MM, Morris MM, Maltais F, Hargreave FE, Pizzichini E. Stable COPD: predicting benefits from high-dose inhaled corticosteroid treatment. Eur Respir J. 2006;27(5):964-971.

13. Brightling CE, Monteiro W, Ward R, et al. Sputum eosinophilia and short-term response to prednisolone in chronic obstructive pulmonary disease: a randomised controlled trial. Lancet. 2000;355(9240): 1480-1485.

14. Brightling CE, Mckenna S, Hargadon B, et al. Sputum eosinophilia and the short term response to inhaled mometasone in chronic obstructive pulmonary disease. Thorax. 2005;60(3):193-198.

15. Pizzichini E, Pizzichini MM, Gibson P, et al. Sputum eosinophilia predicts benefit from prednisolone in smokers with chronic obstructive bronchitis. Am J Respir Crit Care Med. 1998;158(5 Pt 1):1511-1517.

16. Pavord ID, Bafadhel M. Exhaled nitric oxide and blood eosinophilia: independent markers of preventable risk. J Allergy Clin Immunol. 2013;132(4):828-829.

17. Baines KJ, Pavord ID, Gibson PG. The role of biomarkers in the management of airway disease. Int J Tuberc Lung Dis. 2014;18(11): 1264-1268.

18. Pavord ID, Gibson PG. Inflammometry: the current state of play. Thorax. 2012;67(3):191-192.

19. Yap E, Chua WM, Jayaram L, Zeng I, Vandal AC, Garrett J. Can we predict sputum eosinophilia from clinical assessment in patients referred to an adult asthma clinic? Intern Med J. 2013;43(1):46-52.

20. Korevaar DA, Weaterhof GA, Wang J, et al. Diagnostic accuracy of minimally invasive markers for detection of airway eosinophilia in asthma: a systematic review and meta-analysis. Lancet Respir Med. 2015;3(4): 290-300.

21. Westerhof GA, Korevaar DA, Amelink M, et al. Biomarkers to identify sputum eosinophilia in different adult asthma phenotypes. Eur Respir J. 2015;46(3):688-696.

22. Zhang XY, Simpson JL, Powell H, et al. Full blood count parameters for the detection of asthma inflammatory phenotypes. Clin Exp Allergy. 2014;44(9):1137-1145.

23. Fowler SJ, Tavernier G, Niven R. High blood eosinophil counts predict sputum eosinophilia in patients with severe asthma. J Allergy Clin Immunol. 2015;135(3):822-824.

24. Wagener AH, de Nijs SB, Lutter R, et al. External validation of blood eosinophils in asthma. Thorax. 2015;70(2):115-120.

25. Eltboli O, Mistry V, Barker B, Brightling CE. Relationship between blood and bronchial submucosal eosinophilia and reticular basement membrane thickening in chronic obstructive pulmonary disease. Respirology. 2015;20(4):667-670.

26. Bafadhel M, Mckenna S, Terry S, et al. Blood eosinophils to direct corticosteroid treatment of exacerbations of chronic obstructive pulmonary disease: a randomised placebo-controlled trial. Am J Respir Crit Care Med. 2012;186(1):48-55.

27. Pascoe S, Locantore N, Dransfield MT, Barnes NC, Pavord ID Blood eosinophil counts, exacerbations, and response to the addition of inhaled fluticasone foroate to vilanterol in patients with chronic obstructive pulmonary disease: a secondary analysis of data from two parallel randomised controlled trials. Lancet Respir Med. 2015; 3(6):435-442.

28. Siddiqui SH, Guasconi A, Vestbo J, et al. Blood eosinophils: a biomarker of response to extrafine beclomethasone/formoterol in chronic obstructive pulmonary disease. Am J Respir Crit Care Med. 2015; 192(4):523-525

29. Cheng SL, Su KC, Wang HC, Perng DW, Yang PC. Chronic obstructive pulmonary disease treated with inhaled medium- or high-dose corticosteroids: a prospective and randomized study focusing on clinical efficacy and the risk of pneumonia. Drug Des Devel Ther. 2014;8:601-607.

30. Jones PW, Harding G, Berry P, Wiklund I, Chen WH, Kline Leidy N. Development and first validation of the COPD assessment test. Eur Respir J. 2009;34(3):648-654. 
31. Vedel-Krogh S, Nielsen SF, Lange P, Vestbo J, Nordestgaard BG. Blood eosinophils and exacerbations in COPD: the Copenhagen general population study. Am J Respir Crit Care Med. 2016;193(9):965-974.

32. Rahimi-Rad MH, Asgari B, Hosseinzadeh N, Eishi A. Eosinopenia as a marker of outcome in acute exacerbation of chronic obstructive pulmonary disease. Maedica(Buchar). 2015;10(1):10-13.

33. Pavord ID, Lettis S, Locantore N, et al. Blood eosinophils and inhaled corticosteroid/long-acting $\beta$-2 agonist efficacy in COPD. Thorax. 2016;71(2):118-125.

34. Wedzicha JA, Calverley PM, Seemungal TA, Hagan G, Ansari Z, Stockley RA; The INSPIRE investigators. The prevention of chronic obstructive pulmonary disease exacerbations by salmeterol/fluticasone propionate or tiotropium bromide. Am J Respir Crit Care Med. 2008; 177(1):19-26.

35. Calverley P, Pauwels R, Vestbo J, et al; Trial of Inhaled STeroids ANd long-acting beta2 agonists study group. Combined salmeterol and fluticasone in the treatment of chronic obstructive pulmonary disease: a randomised controlled trial. Lancet. 2003;361(9356): 449-456.

36. Zanini A, Cherubino F, Zampogna E, Croce S, Pignatti P, Spanevello A. Bronchial hyperresponsiveness, airway inflammation, and reversibility in patients with chronic obstructive pulmonary disease. Int $J$ Chron Obstruc Pulmon Dis. 2015;10:1155-1161.
37. Iqbal A, Barnes NC, Brooks J. Is blood eosinophil count a predictor of response to bronchodilators in chronic obstructive pulmonary disease? results from post hoc subgroup analyses. Clin Drug Invertig. 2015;35(10):685-688.

38. Schleich FN, Chevremont A, Paulus V, et al. Importance of concomitant local and systemic eosinophilia in uncontrolled asthma. Eur Respir J. 2014;44(1):97-108.

39. Kulkarni NS, Hollins F, Sutcliffe A, et al. Eosinophil protein in airway macrophages: a novel biomarker of eosinophilic inflammation in patients with asthma. J Allergy Clin Immunol. 2010;126(1):61-69.

40. Woodruff PG, Agusti A, Roche N, Singh D, Martinez FJ. Current concepts in targeting chronic obstructive pulmonary disease pharmacotherapy: making progress towards personalised management. Lancet. 2015;385(9979):1789-1798.

41. Ghebre MA, Bafadhel M, Desai D, et al. Biological clustering supports both "Dutch" and "British" hypotheses of asthma and chronic obstructive pulmonary disease. J Allergy Clin Immunol. 2015;135(1):63-72.

42. Brusselle G, Bracke K. Targeting immune pathways for therapy in asthma and chronic obstructive pulmonary disease. Ann Am Thorac Soc. 2014;11(Suppl 5):S322-S328.

\section{Publish your work in this journal}

The International Journal of COPD is an international, peer-reviewed journal of therapeutics and pharmacology focusing on concise rapid reporting of clinical studies and reviews in COPD. Special focus is given to the pathophysiological processes underlying the disease, intervention programs, patient focused education, and self management protocols.

\section{Dovepress}

This journal is indexed on PubMed Central, MedLine and CAS. The manuscript management system is completely online and includes a very quick and fair peer-review system, which is all easy to use. Visit $\mathrm{http} / / / \mathrm{www}$.dovepress.com/testimonials.php to read real quotes from published authors. 